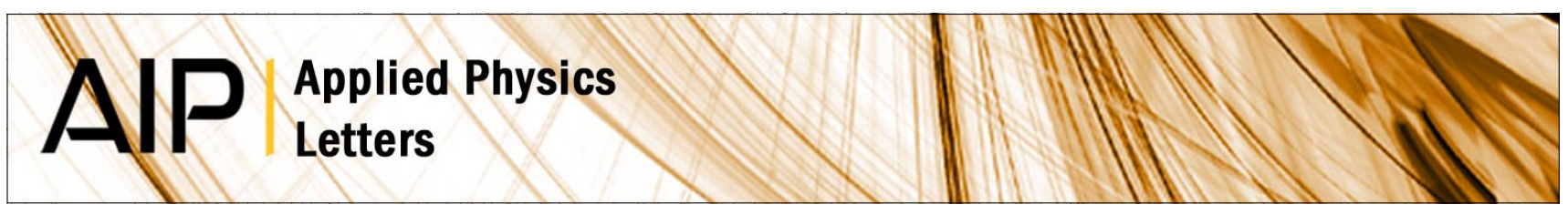

\title{
Lone conduction band in Cu2ZnSnSe4
}

Levent Gütay, Alex Redinger, Rabie Djemour, and Susanne Siebentritt

Citation: Appl. Phys. Lett. 100, 102113 (2012); doi: 10.1063/1.3691945

View online: http://dx.doi.org/10.1063/1.3691945

View Table of Contents: http://apl.aip.org/resource/1/APPLAB/v100/i10

Published by the American Institute of Physics.

\section{Related Articles}

Band gap control via tuning of inversion degree in Cdln2S4 spinel

Appl. Phys. Lett. 100, 102112 (2012)

Direct imaging and optical activities of stacking faults in $4 \mathrm{H}-\mathrm{SiC}$ homoepitaxial films

J. Appl. Phys. 111, 053513 (2012)

Steady state and transient electron transport properties of bulk dilute GaNxAs1-x

J. Appl. Phys. 111, 053703 (2012)

Efficient algorithm of the transcorrelated method for periodic systems

J. Chem. Phys. 136, 094108 (2012)

Valence band structure in boron-zinc oxide films characterized by secondary electron emission

J. Appl. Phys. 111, 053302 (2012)

\section{Additional information on Appl. Phys. Lett.}

Journal Homepage: http://apl.aip.org/

Journal Information: http://apl.aip.org/about/about_the_journal

Top downloads: http://apl.aip.org/features/most_downloaded

Information for Authors: http://apl.aip.org/authors

\section{ADVERTISEMENT}

NEW!

iPeerReview

AlP's Newest App

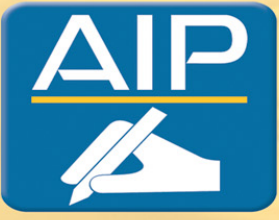

Authors...

Reviewers...

Check the status of

submitted papers remotely!

ADPublishing 


\title{
Lone conduction band in $\mathrm{Cu}_{2} \mathrm{ZnSnSe}_{4}$
}

\author{
Levent Gütay, ${ }^{\text {a) }}$ Alex Redinger, Rabie Djemour, and Susanne Siebentritt \\ Laboratory for Photovoltaics, University of Luxembourg, L-4422 Belvaux, Luxembourg
}

(Received 5 January 2012; accepted 16 February 2012; published online 9 March 2012)

\begin{abstract}
We present experimental proof for a narrow first conduction band in $\mathrm{Cu}_{2} \mathrm{ZnSnSe}_{4}$ semiconductor films as it has been predicted by theoretical calculations. The optical absorption characteristics of $\mathrm{Cu}_{2} \mathrm{ZnSnSe}_{4}$ thin films are analyzed by optical transmission spectroscopy. The experimental data show strong evidence for three absorption edges, as expected from theory, involving the valence band and two conduction bands, which are separated by a second band gap. (C) 2012 American Institute of Physics. [http://dx.doi.org/10.1063/1.3691945]
\end{abstract}

The kesterite compound semiconductors $\mathrm{Cu}_{2} \mathrm{ZnSnSe}_{4}$ (CZTSe) and $\mathrm{Cu}_{2} \mathrm{ZnSnS}_{4}$ (CZTS) are promising candidates for the application as absorbers in thin film solar cells, with efficiencies exceeding $10 \%$. These compounds are, in comparison to current high efficiency thin film materials as $\mathrm{Cu}(\mathrm{In}, \mathrm{Ga}) \mathrm{Se}_{2}$ and $\mathrm{CdTe}$, composed of elements of higher abundance and less toxicity. ${ }^{1-3}$

All theoretical investigations of the band structures of CZTSe and CZTS show a peculiar density of states in the conduction band: ${ }^{4-8}$ The conduction band minimum is formed by a rather narrow band, formed by Sn-s and anion-p states. At higher energies, the density of states becomes zero again, forming a second band gap within the conduction band. For CZTSe, the reports show a direct band gap $\mathrm{E}_{\mathrm{g} 1}$ between the valence band (VB) and the first conduction band (CB1) in the range of $1 \mathrm{eV} .^{6,7}$ The second bandgap $\mathrm{E}_{\mathrm{g} 2}$ between the first narrow conduction band and the higher bands in the conduction band is in the range $0.5-1.5 \mathrm{eV}$; the width of $\mathrm{CB} 1$, which remains a lone conduction band, is calculated to be $\mathrm{CB} 1_{\text {width }} \approx 0.7-1.2 \mathrm{eV} .^{6,7}$ In principle, such a band structure can be expected to show three optical transitions at $\mathrm{E}_{\mathrm{g} 1}, \mathrm{E}_{\mathrm{g} 2}$, and $\mathrm{E}_{\mathrm{g} 3}=\mathrm{E}_{\mathrm{g} 1}+\mathrm{E}_{\mathrm{g} 2}+\mathrm{CB} 1_{\text {width }}$. Considering the theoretical $\mathrm{E}(\mathrm{k})$ dispersion curves from literature, ${ }^{6,7}$ it is not unambiguously clear if $\mathrm{E}_{\mathrm{g} 2}$ and $\mathrm{E}_{\mathrm{g} 3}$ are direct or indirect. So far, experimental results are reported only for the first absorption edge around $1 \mathrm{eV} .^{9-12}$ In this manuscript, we report on our findings on the absorption behavior of CZTSe absorber films above this photon energy and present experimental results which confirm the existence of a lone conduction band.

In a semiconductor containing a lone first conduction band, the absorption coefficient can be expected to decrease to some extent at photon energies above $\mathrm{E}_{\mathrm{g} 1}+\mathrm{CB} 1_{\text {width }}$ and/

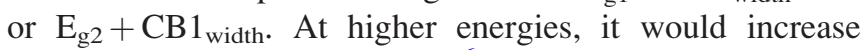
again due to the transition $\mathrm{E}_{\mathrm{g} 3}{ }^{6}$ As absorber layers of the usual thickness (i.e., $>1 \mu \mathrm{m}$ ) do not transmit any measureable light above the first band gap, for the purpose of this study, we have grown thin CZTSe absorber films which allow for studies by optical transmission. The samples have been prepared via a two stage process. First, a precursor layer is co-evaporated from $\mathrm{Zn}, \mathrm{Sn}$, and $\mathrm{Cu}$ effusion cells,

\footnotetext{
${ }^{\text {a) }}$ Author to whom correspondence should be addressed. Electronic mail: levent.guetay@uni.lu.
}

and Se is supplied from a valved source. The deposition has been carried onto soda lime glass, and the deposition temperature has been set to $320^{\circ} \mathrm{C}$. This procedure enables a thorough control of the film composition and eliminates reevaporation of SnSe. ${ }^{13}$ In a second step, the films are postannealed in a tube furnace in order to improve the crystal quality. A temperature of $500^{\circ} \mathrm{C}$ has been chosen, and the annealing period has been set to $30 \mathrm{~min}$. The annealing has been carried out in a $\mathrm{H}_{2} / \mathrm{N}_{2}$ gas mixture (1 mbar total pressure), and additional Se and SnSe have been introduced into the hot zone in order to prevent the decomposition of the absorber layer, described in detail elsewhere. ${ }^{3,14}$ The duration of the growth step has been adapted to achieve a film thickness of approximately $200 \mathrm{~nm}$ and $400 \mathrm{~nm}$. For thicker samples, which are otherwise grown by the same procedure as the samples discussed here, solar cell efficiencies higher than $4 \%$ have been achieved.

The presence of the kesterite phase was assessed by photoluminescence (PL) and Raman, at which no indication for a contribution of secondary phases was visible. The measured spectra with a PL peak at $\approx 0.95 \mathrm{eV}$ and the main Raman shift at $\approx 195 \mathrm{~cm}^{-1}$ fit the acknowledged kesterite fingerprints in literature, respectively. ${ }^{10,11,15}$

The absorption behavior of the samples is determined by optical transmission measurements which were performed in an UV-VIS spectrometer with an Ulbricht sphere. The recorded optical transmission data were evaluated by an optical modeling tool kit in the program Diplot. ${ }^{16}$ This modeling includes the optical absorption behavior of the absorber material as such and the geometrical properties and structure of the sample. Diplot allows to determine the absorption behaviour from a measurement of the transmission alone, without measuring the reflection. The model describes the reflection using the refractive index, which is fitted to the interference behavior in the transparent region. The full modeling is also necessary to distinguish if peaks and other features of the measured transmission spectra occur from Fabry-Perot-interferences of the transmitted light or from the actual behavior of the absorption coefficient. The absorption is modeled by band-band transitions either for a direct or an indirect transition. The imperfection of the crystal is taken into account by adding Urbach tailing to the density of states.

In Fig. 1, we show the optical transmission spectrum of the $\approx 200 \mathrm{~nm}$ thick CZTSe film. The blue solid curve shows 


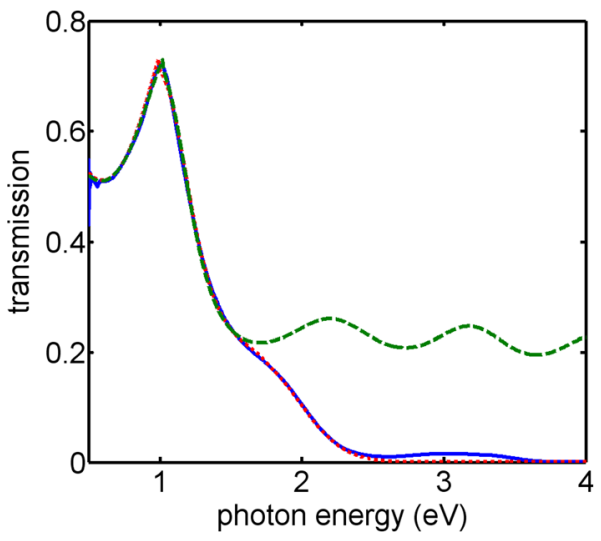

FIG. 1. (Color online) Measurement (blue solid curve) and modeling of optical transmission of $\approx 190 \mathrm{~nm}$ thick CZTSe layer. Models include i) a direct $\left(\mathrm{E}_{\mathrm{g}}=1 \mathrm{eV}\right.$ ) band gap (dashed green curve) and ii) a direct plus an indirect band gap $\left(\mathrm{E}_{\mathrm{g} 1}=1 \mathrm{eV}, \mathrm{E}_{\mathrm{g} 2}=1.6 \mathrm{eV}\right)$ (red dotted curve).

the actual measurement, and the dashed green and dotted red ones are modeling results calculated with Diplot. The observation of the measured spectrum shows one Fabry-Perot oscillation with its minimum and maximum at $\approx 0.6 \mathrm{eV}$ and $\approx 1 \mathrm{eV}$, respectively. This oscillation is fitted best for a modeled sample thickness of $\approx 190 \mathrm{~nm}$, which matches the estimation from the growth rate of the deposition process well. For the modeling, a refractive index of $\mathrm{n} \approx 2.8$ is used for the respective spectral region, fitting the intensity of the oscillation best. ${ }^{17}$ The measured curve shows a steep decrease of the transmission signal at photon energies of $1-1.5 \mathrm{eV}$ and a more shallow behavior at $1.5-2.5 \mathrm{eV}$. The signal shows a minimum at $2.6 \mathrm{eV}$, then an increase reaching a shallow maximum at $\approx 3 \mathrm{eV}$, and a final decrease with full extinction at above $3.6 \mathrm{eV}$.

The green dashed curve in Figure 1 shows a modeling approach which includes only a single direct band gap $\mathrm{E}_{\mathrm{g}}=1 \mathrm{eV}$, as has been reported before. ${ }^{9-12}$ The absorption coefficient is $\alpha>10^{4} \mathrm{~cm}^{-1}$ at photon energies $>1.05 \mathrm{eV}$, and Urbach energy is $\mathrm{E}_{\mathrm{Ur}} \approx 170 \mathrm{meV}$. The calculated curve fits the measured spectrum well in the range $<1.4 \mathrm{eV}$. Above $1.4 \mathrm{eV}$, the modeled curve shows a remaining transmission which oscillates around $0.2-0.25$, as the given layer thickness is not enough to absorb the light fully. This striking discrepancy between model and experiment is not visible with thick layers $(\mathrm{d}>1 \mu \mathrm{m})$, where light above the band gap is completely absorbed. The result of this single gap model shows unequivocally that the absorption behavior of CZTSe cannot be explained by only a single band gap.

The dotted red curve in Figure 1 shows the result of a two band gap modeling approach, including a direct band gap $\mathrm{E}_{\mathrm{g} 1}=1 \mathrm{eV}$ and an indirect one $\mathrm{E}_{\mathrm{g} 2}=1.6 \mathrm{eV}$. The fit to the experimental transmission spectrum is a lot better than assuming the second band gap to be direct. These two gaps correspond to the transitions described as $\mathrm{E}_{\mathrm{g} 1}$ and $\mathrm{E}_{\mathrm{g} 2}$ in the above discussion of the theoretical background. Further parameters of the two modeled transitions are $\alpha_{1}>10^{4} \mathrm{~cm}^{-1}$ at photon energies $>1.05 \mathrm{eV}$ with $\mathrm{E}_{\mathrm{Ur} 1} \approx 170 \mathrm{meV}$ and $\alpha_{2}>10^{4} \mathrm{~cm}^{-1}$ at photon energies $>1.66 \mathrm{eV}$ with $\mathrm{E}_{\mathrm{Ur} 2} \approx 60 \mathrm{meV}$, respectively. The curve fits the measured spectrum well in the range $<2.3 \mathrm{eV}$. Above $2.3 \mathrm{eV}$, the measured curve shows a less steep decrease than the simulation and the transmission maximum in the

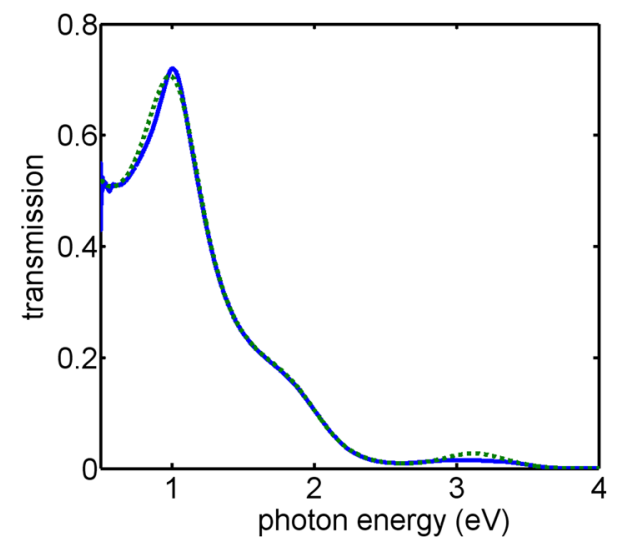

FIG. 2. (Color online) Measurement (blue solid) and modeling (green, dotted) of optical transmission spectrum of $\approx 190 \mathrm{~nm}$ thick CZTSe layer. Model includes two absorption bands for transitions at $\mathrm{E}_{\mathrm{g} 1}$ and $\mathrm{E}_{\mathrm{g} 2}$ and an additional indirect band gap $\mathrm{E}_{\mathrm{g} 3}=2.85 \mathrm{eV}$.

$3 \mathrm{eV}$ region. At this photon energy, the modeled curve shows a full extinction of the transmission. This departure can be explained as follows: As discussed above, a lone first conduction band can cause a decrease of the absorption to some extent at photon energies above $\mathrm{E}_{\mathrm{g} 1}+\mathrm{CB} 1_{\text {width }}$ and/or $\mathrm{E}_{\mathrm{g} 2}+\mathrm{CB} 1_{\text {width. }}$ The transitions in this modeling approach do not account for such a decrease. The modeled absorption coefficients increase as a function of the photon energy consistently with square-root or square behavior for direct or indirect band gaps, i.e., $E \cdot \alpha \propto\left(E-E_{g}\right)^{X}$, with $\mathrm{X}=0.5$ or 2 , respectively. This two-band-gap model is, therefore, only able to conclusively describe the measured transmission as long as the absorption coefficient of both transitions increases with this behavior, which is not the case for the higher energy region. For the spectral region above $2.3 \mathrm{eV}$, this behavior seems to change as expected for a lone conduction band, calling for a further modification of the model.

The third approach includes two absorption bands to simulate the absorption at $\mathrm{E}_{\mathrm{g} 1}$ and $\mathrm{E}_{\mathrm{g} 2}$. The two absorption bands are represented by broad absorption peaks of Lorentz shape. This approach accounts for the narrowness of the first conduction band and allows for simulating a decreasing absorption coefficient for higher photon energies, resulting in an increasing transmission signal for higher energies. The decrease of the transmission above $3.2 \mathrm{eV}$ is then fitted with an indirect third band gap with $\mathrm{E}_{\mathrm{g} 3}=2.85 \mathrm{eV}$. Figure 2 shows the modeling result in comparison to the measured spectrum. In this last approach, the main emphasis is put on modeling the higher energy region of the transmission spectrum to extract a band gap value for $E_{\mathrm{g} 3}$. The lower energy part of the spectrum, particularly the region at $<1.3 \mathrm{eV}$, is only roughly reproduced by the approach via the Lorentz shaped absorption bands, which may not be the best approach for the description of the absorption by the lone band. However, it allows to describe the main features of the observed transmission spectrum. Further, the comparison of the plots shows a slight difference in the height of the feature in the $3 \mathrm{eV}$ region. This can be explained by the fact that the model does not explicitly consider transitions from deeper energy levels of the valence band into the first conduction band and transitions from the first conduction band into higher levels of the upper conduction bands. This is 


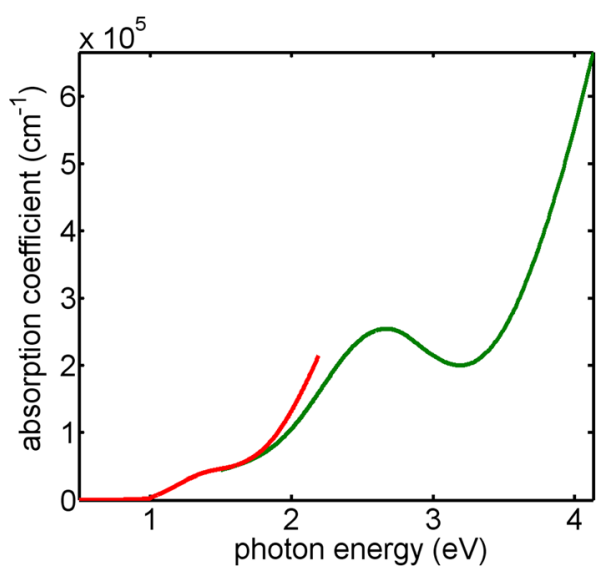

FIG. 3. (Color online) Absorption coefficient extracted from the two band gap (low energy part, red) and three band gap (high energy part, green) modeling approach. Each curve is plotted only for the spectral region of validity for the respective model.

effectively only very roughly approximated by the width and tailing of the fitted Lorentz shaped absorption peaks. Therefore, the spectral feature at $\approx 3 \mathrm{eV}$ shows a slight difference between model and experiment for the transmission signal.

In Figure 3, we show the spectral absorption coefficient extracted from the modeling. The low energy part (red curve) is extracted from the two band gap approach and the high energy part (green curve) from the three band gap fit. Each curve is plotted only for the spectral region where it can be regarded as valid, as it was discussed above. The shape and height of the spectrum show strong similarity with the theoretically predicted spectral absorption coefficient by Persson. ${ }^{6}$ The onset energies for the increase of absorption at the first and second band gap and the positions of the maximum and minimum at $\approx 2.6 \mathrm{eV}$ and $\approx 3.2 \mathrm{eV}$, respectively, show a fair agreement.

In Figure 4, we show additional measurements on an as grown sample with $\approx 380 \mathrm{~nm}$ thickness and a sample with $\approx 120 \mathrm{~nm}$ thickness which was prepared by thinning with a bromine-methanol etching. In the $380 \mathrm{~nm}$ case, the maximum in the $3 \mathrm{eV}$ region is only very slightly visible, as almost all light above $2.1 \mathrm{eV}$ is absorbed at this sample thickness. The inset is a zoom into the region around $3 \mathrm{eV}$, showing that the position of the spectral feature stays approximately the same for all sample thicknesses. This additionally supports that this feature is related to decreased absorption and not to

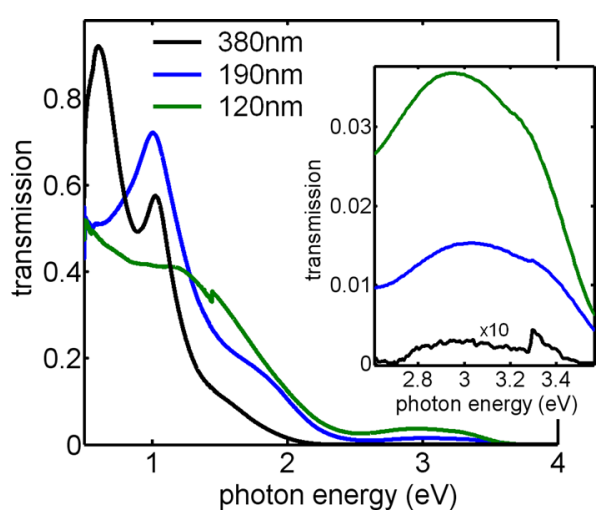

FIG. 4. (Color online) Transmission spectrum for different thicknesses $d$. Inset shows zoom into $3 \mathrm{eV}$ region.
Fabry-Perot oscillations or other artifacts of the measurement. Fitting results for these additional spectra (not shown here) support the quantitative results for the discussed transitions.

In this manuscript, we have experimentally shown that three band gaps are present in the absorption spectrum of kesterite CZTSe absorbers. The experimental data were fitted by a model including three transitions, i.e., a direct band gap $\mathrm{E}_{\mathrm{g} 1}=1 \mathrm{eV}$ between the valence band and the first conduction band, an indirect band gap $\mathrm{E}_{\mathrm{g} 2}=1.6 \mathrm{eV}$ between the first and the second conduction band, and another indirect transition $\mathrm{E}_{\mathrm{g} 3}=2.85 \mathrm{eV}$ between the valence band and the second conduction band. These results confirm the theoretical expectation of a lone first conduction band as was recently reported. ${ }^{6,7}$ In addition, they demonstrate that the second and third band gaps are indirect, i.e., the minimum of the second conduction band is not at the $\Gamma$ point. In contrast to the calculated densities of state, our results indicate a slightly larger gap $\mathrm{E}_{\mathrm{g} 2}$ and a more narrow first conduction band with $\mathrm{CB} 1_{\text {width }}=\mathrm{E}_{\mathrm{g} 3}-\mathrm{E}_{\mathrm{g} 2}-\mathrm{E}_{\mathrm{g} 1} \approx$ $0.25 \mathrm{eV}$. Here, we have to state that for the modeling of each transition, we assume the transition to begin at the maximum of the lowest band that is involved. This is of course true for the transitions $\mathrm{E}_{\mathrm{g} 1}$ and $\mathrm{E}_{\mathrm{g} 3}$. For $\mathrm{E}_{\mathrm{g} 2}$, the situation is a bit more complicated: The charge carriers in $\mathrm{CB} 1$ are relaxing to the thermal distribution which is determined by the Fermi-Dirac-distribution and the ambient temperature. Therefore, we assume that there are enough charge carriers present in the entire band $\mathrm{CB} 1$. According to this argumentation also the transition $\mathrm{E}_{\mathrm{g} 2}$ can be assumed to start at the maximum of the lower one of the involved bands (CB1). Still, we have to admit that it could be possible that our approach overestimates $\mathrm{E}_{\mathrm{g} 2}$ by the amount of a few $\mathrm{k}_{\mathrm{B}} \mathrm{T}$ and underestimates $\mathrm{CB} 1_{\text {width }}$ by the same amount, as the effective onset energy of this transition can be expected to depend strongly on the charge carrier profile and concentration in CB1. Concluding this error analysis, we can state that these discussed effects do not affect the major findings and conclusions in this letter.

The minimum in absorption is still at a high absorption coefficient of about $2 \times 10^{5} \mathrm{~cm}^{-1}$. It can, therefore, be expected that it does not influence the efficiency of solar cells. Besides, it occurs at energies higher than the band gap of CdS which is used as a buffer layer in these solar cells; thus, light of this energy is already being absorbed in the buffer. However, the experimental finding that the lone band is even narrower than predicted by the calculations indicates a flatter dispersion and thus a quite high effective mass of the electrons, which can limit their diffusion length. Furthermore, the predicted and experimentally confirmed density of states with a large band $\mathrm{E}_{\mathrm{g} 3}$ and an intermediate band within the gap is the structure sought for the application in intermediate band solar cells. ${ }^{18}$

This work was supported by TDK Corporation in the framework of the TDK-Europe professorship and by the Luxembourgish Fonds National de la Recherche in the framework of the KITS project (No. C08/MS/20). Monika Arasimowicz is acknowledged for technical assistance during etching.

${ }^{1}$ D. A. R. Barkhouse, O. Gunawan, T. Gokmen, T. K. Todorov, and D. B. Mitzi, Prog. Photovoltaics 20(1), 6 (2012).

${ }^{2}$ Q. Guo, G. M. Ford, W.-C. Yang, B. C. Walker, E. A. Stach, H. W. Hillhouse, and R. Agrawal, J. Am. Chem. Soc. 132(49), 17384 (2010). 
${ }^{3}$ A. Redinger, D. M. Berg, P. J. Dale, R. Djemour, L. Gütay, T. Eisenbarth, N. Valle, and S. Siebentritt, IEEE J. Photovoltaics 1(2), 200 (2011).

${ }^{4}$ J. Paier, R. Asahi, A. Nagoya, and G. Kresse, Phys. Rev. B 79, 115126 (2009).

${ }^{5}$ S. Chen, X. G. Gong, A. Walsh, and S.-H. Wei, Appl. Phys. Lett. 94, 041903 (2009).

${ }^{6}$ C. Persson, J. Appl. Phys. 107, 053710 (2010).

${ }^{7}$ S. Botti, D. Kammerlander, and M. A. L. Marques, Appl. Phys. Lett. 98, 241915 (2011).

${ }^{8}$ S. Siebentritt and S. Schorr, "Kesterites—a challenging material for solar cells," Prog. Photovoltaics (in press).

${ }^{9}$ S. Ahn, S. Jung, J. Gwak, A. Cho, K. Shin, K. Yoon, D. Park, H. Cheong, and J. H. Yun, Appl. Phys. Lett. 97(2), 021905 (2010).

${ }^{10}$ P. M. P. Salomé, P. A. Fernandes, A. F. d. Cunha, J. P. Leitão, J. Malaquias, A. Weber, J. C. González, and M. I. N. d. Silva, Sol. Energy Mater. Sol. Cells 94(12), 2176 (2010).
${ }^{11}$ M. Grossberg, J. Krustok, K. Timmo, and M. Altosaar, Thin Solid Films 517(7), 2489 (2009).

${ }^{12}$ D. Park, D. Nam, S. Jung, S. An, J. Gwak, K. Yoon, J. H. Yun, and H. Cheong, Thin Solid Films 519, 7386 (2011).

${ }^{13}$ A. Redinger and S. Siebentritt, Appl. Phys. Lett. 97(9), 092111 (2010).

${ }^{14}$ A. Redinger, D. M. Berg, P. J. Dale, and S. Siebentritt, J. Am. Chem. Soc. 133, 3320 (2011).

${ }^{15}$ A. Redinger, K. Hönes, X. Fontané, V. Izquierdo-Roca, E. Saucedo, N. Valle, A. Pérez-Rodríguez, and S. Siebentritt, Appl. Phys. Lett. 98(10), 101907 (2011).

${ }^{16}$ E. Lotter (E. Lotter, Besigheim, Germany, 2009), DIPLOT, see www.diplot.de.

${ }^{17}$ The refractive index $\mathrm{n}$ is additionally confirmed by fitting transmission spectra from thicker absorbers of which the thickness was measured by electron microscopy.

${ }^{18}$ A. Luque and A. Marti, Phys. Rev. Lett. 78(26), 5014 (1997). 